\title{
Inverse Boundary Value Problem for the Magnetohydrodynamics Equations
}

\author{
Lianhua He $\mathbb{D}^{1,2}$ and Zhong Tan $\mathbb{D}^{3}$ \\ ${ }^{1}$ School of Mathematics Science, Guizhou Normal University, Guiyang 550001, China \\ ${ }^{2}$ School of Mathematical Sciences, Xiamen University, Xiamen 361005, China \\ ${ }^{3}$ School of Mathematical Sciences and Fujian Provincial Key Laboratory on Mathematical Modeling and Scientific Computing, \\ Xiamen University, Xiamen 361005, China
}

Correspondence should be addressed to Zhong Tan; tan85@xmu.edu.cn

Received 22 March 2021; Accepted 12 June 2021; Published 21 June 2021

Academic Editor: Maria Alessandra Ragusa

Copyright (C) 2021 Lianhua He and Zhong Tan. This is an open access article distributed under the Creative Commons Attribution License, which permits unrestricted use, distribution, and reproduction in any medium, provided the original work is properly cited.

In this paper, we consider the stationary magnetohydrodynamics (MHD) equations in a bounded domain of $\mathbb{R}^{d}(d=2,3)$ with viscosity and magnetic diffusing. By the linearization technique, we prove that the uniqueness of viscosity function and magnetic diffusing function in the MHD equations is determined from the knowledge of the Cauchy data measured on the boundary.

\section{Introduction}

Let $\Omega \subset \mathbb{R}^{d}(d=2,3)$ be an open bounded domain with boundary $\partial \Omega \in C^{\infty}$. Assume that $\Omega$ is filled with an incompressible fluid. $u \in \mathbb{R}^{d}$ and $B \in \mathbb{R}^{d}$ stand for velocity field and magnetic field, respectively. $(u, B)$ satisfies the following stationary magnetohydrodynamics(MHD) equations:

$$
\begin{cases}\operatorname{div}\left(\sigma_{\mu}(u, p)\right)-u \cdot \nabla u+B \cdot \nabla B=0 & \text { in } \Omega, \\ \operatorname{div} u=0 & \text { in } \Omega, \\ \operatorname{div}\left(\tilde{\sigma}_{\lambda}(B)\right)-u \cdot \nabla B+B \cdot \nabla u=0 & \text { in } \Omega, \\ \operatorname{div} B=0 & \text { in } \Omega .\end{cases}
$$

where

$$
\sigma_{\mu}(u, p)=2 \mu \operatorname{Sym}(\nabla u)-p I ; \tilde{\sigma}_{\lambda}(B)=2 \lambda \operatorname{Sym}(\nabla B),
$$

and $\operatorname{Sym}(A)=\left(A+A^{T}\right) / 2$ is the symmetric part of the matrix $A$. $p$ is the pressure. The notation $I$ is the identity matrix. Here, $\mu(x)>0$ is the viscosity function and $\lambda(x)>0$ is the magnetic diffusivity function.
In this paper, we are interested in the inverse problem for MHD equation. First, we define the Cauchy data for the MHD equation (1) by

$$
\tilde{C}_{\mu, \lambda}=\left\{\left(\left.u\right|_{\partial \Omega},\left.B\right|_{\partial \Omega},\left.\sigma_{\mu}(u, p) \mathbf{n}\right|_{\partial \Omega},\left.\tilde{\sigma}_{\lambda}(B) \mathbf{n}\right|_{\partial \Omega}\right) \mid(u, p, B) \text { satisfies the equations }\right\},
$$

where $\mathbf{n}$ is the unit outer normal of the boundary $\partial \Omega$ and $\left.u\right|_{\partial \Omega}$, and $\left.B\right|_{\partial \Omega}$ satisfies the compatibility conditions

$$
\left.\int_{\partial \Omega} u\right|_{\partial \Omega} \cdot \mathbf{n} d s=0,\left.\int_{\partial \Omega} B\right|_{\partial \Omega} \cdot \mathbf{n} d s=0
$$

In the physical sense, $\left.\sigma_{\mu}(u, p) \mathbf{n}\right|_{\partial \Omega}$ stands for the stress acting on $\partial \Omega$ and is called the Cauchy force. The motivation of this paper is to determine $(\mu, \lambda)$ from the knowledge of the Cauchy data $\tilde{C}_{\mu, \lambda}$.

To discuss the inverse problem, we will not consider the general Dirichlet data $\left.(u, B)\right|_{\partial \Omega}=\left(\phi_{1}, \phi_{2}\right)$. Particularly, we shall assume

$$
\left(\phi_{1}, \phi_{2}\right)=\left(\varepsilon \psi_{1}, \varepsilon \psi_{2}\right)
$$


with $|\varepsilon|$ sufficiently small and $\psi_{i} \in H^{3 / 2}(\partial \Omega)(i=1,2)$ satisfying the compatibility condition (4). For such a choice of Dirichlet data, we can obtain that there exists a solution $(u, p, B)$ of the equation (1) with $\left.u\right|_{\partial \Omega}=\varepsilon \psi_{1},\left.B\right|_{\partial \Omega}=\varepsilon \psi_{2}$ and the boundary trace $\left.\sigma_{\mu}(u, p) \mathbf{n}\right|_{\partial \Omega},\left.\tilde{\sigma}_{\lambda}(B) \mathbf{n}\right|_{\partial \Omega} \in H^{1 / 2}(\partial \Omega)$. Thus, the Cauchy data $\tilde{C}_{\mu, \lambda}$ is meaningful in this case. When $|\varepsilon|$ is sufficiently small, we even know that the solution $(u, p, B)$ to (1) is unique ( $p$ is unique up to a constant), but we do not need it. The main results of this paper are the following global uniqueness theorems of the inverse problem.

Theorem 1. Let $\Omega \subset \mathbb{R}^{3}$. Assume that $\mu_{1}(x)$ and $\mu_{2}(x)$ are viscosity functions and $\lambda_{1}(x)$ and $\lambda_{2}(x)$ are magnetic diffusivity functions. $\mu_{i}, \lambda_{i} \in C^{n_{0}}(\bar{\Omega})(i=1,2)$ for $n_{0} \geq 8$ and

$$
\begin{aligned}
& \partial^{\alpha} \mu_{1}(x)=\partial^{\alpha} \mu_{2}(x) \forall x \in \partial \Omega,|\alpha| \leq 1, \\
& \partial^{\beta} \lambda_{1}(x)=\partial^{\beta} \lambda_{2}(x) \forall x \in \partial \Omega,|\beta| \leq 1 .
\end{aligned}
$$

Let $\tilde{C}_{\mu_{1}, \lambda_{1}}$ and $\tilde{C}_{\mu_{2}, \lambda_{2}}$ be the Cauchy data with $\left(\mu_{1}, \lambda_{1}\right)$ and $\left(\mu_{2}, \lambda_{2}\right)$, respectively. If $\tilde{C}_{\mu_{1}, \lambda_{1}}=\tilde{C}_{\mu_{2}, \lambda_{2}}$, then $\left(\mu_{1}, \lambda_{1}\right)=\left(\mu_{2}, \lambda_{2}\right)$.

When the boundary $\partial \Omega$ is convex and has nonvanishing Gauss curvature, we can remove the assumption (6)(7) from Theorem 1.

Theorem 2. Let $\Omega \subset \mathbb{R}^{3}$. Suppose that $\partial \Omega$ is convex with the nonvanishing Gauss curvature, and $\mu_{i}(x)$ and $\lambda_{i}(x)(i=1,2)$ are viscosity functions and magnetic diffusivity functions, respectively, satisfying $\mu_{i}, \lambda_{i} \in C^{n_{0}}(\bar{\Omega})$ for $n_{0} \geq 8$. If $\tilde{C}_{\mu_{1}, \lambda_{1}}=$ $\tilde{C}_{\mu_{2}, \lambda_{2}}$, then $\left(\mu_{1}, \lambda_{1}\right)=\left(\mu_{2}, \lambda_{2}\right)$.

Theorem 3. Let $\Omega$ be a simply connected bounded domain in $\mathbb{R}^{2}$ with smooth boundary. Suppose that $\mu_{i}(x)$ and $\lambda_{i}(x)$ $(i=1,2)$ are viscosity functions and magnetic diffusivity functions, respectively. Assume that $\mu_{i}(x), \lambda_{i}(x) \in C^{3}(\bar{\Omega})$ and $\mu_{i}(x), \lambda_{i}(x)>0$ with

$$
\begin{aligned}
& \left.\partial^{\alpha} \mu_{1}\right|_{\partial \Omega}=\left.\partial^{\alpha} \mu_{2}\right|_{\partial \Omega} \text { for all }|\alpha| \leq 1, \\
& \left.\partial^{\beta} \lambda_{1}\right|_{\partial \Omega}=\left.\partial^{\beta} \lambda_{2}\right|_{\partial \Omega} \text { for all }|\beta| \leq 1 .
\end{aligned}
$$

Let $\tilde{C}_{\mu_{1}, \lambda_{1}}$ and $\tilde{C}_{\mu_{2}, \lambda_{2}}$ be the Cauchy data associated with $\left(\mu_{1}, \lambda_{1}\right)$ and $\left(\mu_{2}, \lambda_{2}\right)$, respectively. If $\tilde{C}_{\mu_{1}, \lambda_{1}}=\tilde{C}_{\mu_{2}, \lambda_{2}}$, then $\left(\mu_{1}, \lambda_{1}\right)=\left(\mu_{2}, \lambda_{2}\right)$.

If we attempt to determine the internal parameters of body, we can make measurements only at the surface of the body. This is the well-known inverse boundary value problems. A typical application of the inverse problem is electrical impedance tomography (EIT). Since the 1980s, the parameter determination problem by boundary measurements has been well studied. Since Calderón's pioneer contribution [1], the method of complex geometrical optics solutions (see [2-4]) which was introduced by Sylvester and Uhlmann
[5] has become a standard method. There are other methods to research the inverse boundary value problems, for example, by using the Dirichlet-to-Neumann map (see [6-8]), complex exponential solution (see [9-11]), and Cauchy data $([12,13])$. The global uniqueness of identifying the viscosity using the Cauchy data is a rather well-studied field. For the Stokes equation, the uniqueness for the inverse boundary problem was considered by Heck et al. [9] and Lai et al. [12]. The unique determination of the viscosity $\mu$ for the Navier-Stokes equations is proved by $\mathrm{Li}$ and Wang [14] in dimension three, Imanuvilov and Yamamoto [7] in dimension two, and Lai et al. [12] in dimension two. In [12, 1416], they used the linearization method to study the uniqueness determination of $\mu$ for the Navier-Stokes equations. This method was first introduced by Isakov [17] in a semilinear parabolic inverse problem. This technique allows for the reduction of the semilinear inverse boundary value problem to the corresponding linear one.

In this paper, we would like to apply the linearization technique to consider the unique determination problem of parameters in MHD equations. The main difficulty in applying the linearization technique to solve our problem lies in the existence of particular solutions to (1) which has some controlled asymptotic properties. In order to solve this difficulty, we only consider the Dirichlet condition with small parameter $\varepsilon$ as in (5). The key step in the proofs of Theorems 1,2 , and 3 is to prove the existence of the solution $\left(u^{\varepsilon}, p^{\varepsilon}, B^{\varepsilon}\right)$ to (1) with boundary condition (5) and $\left(\varepsilon^{-1} u^{\varepsilon}, \varepsilon^{-1} p^{\varepsilon}, \varepsilon^{-1} B^{\varepsilon}\right)$ converging to $\left(u_{0}, p_{0}, B_{0}\right)$ in suitable Sobolev spaces, where $\left(u_{0}, p_{0}\right)$ and $B_{0}$ satisfy two Stokes equations, respectively, whereafter the Cauchy data $\tilde{C}_{\mu, \lambda}$ of MHD equation can determine Cauchy data $C_{\mu}$ of the Stokes equation about $u$ and $C_{\lambda}$ of the Stokes equation about $B$. Then, the inverse problem for the MHD equation (1) is reduced to the same problem for the Stokes equation. The innovation point in this paper is the reduction of the nonlinear inverse boundary value problem to two corresponding ones.

This paper is organized as follows. In Section 2, we will prove the existence of the boundary value problem for (1). In Section 3, we linearize the Cauchy data $\tilde{C}_{\mu, \lambda}$ and prove Theorems 1, 2, and 3.

\section{Direct Problem}

In this section, we would like to prove the existence of the boundary value problem:

$$
\begin{cases}\operatorname{div}\left(\sigma_{\mu}(u, p)\right)-u \cdot \nabla u+B \cdot \nabla B=0 & \text { in } \Omega, \\ \operatorname{div}\left(\tilde{\sigma}_{\lambda}(B)\right)-u \cdot \nabla B+B \cdot \nabla u=0 & \text { in } \Omega, \\ \operatorname{div} u=0, \operatorname{div} B=0 & \text { in } \Omega, \\ u=\phi_{1}, B=\phi_{2} & \text { on } \partial \Omega,\end{cases}
$$

with $\phi_{-} i(i=1,2) \in H^{3 / 2}(\partial \Omega)$ and the compatibility conditions (4). When $\mu$ and $\lambda$ are constants, this problem has been discussed in literature [18]. 
In order to prove the existence of equation (9), we first introduce some lemmas.

Lemma 4 (see [14], Theorem 10). Suppose $\mu \in C^{1,1}(\bar{\Omega})$. For any,

$$
f \in W^{-1, s}(\Omega), g \in L^{s}(\Omega), \psi \in W^{1-1 / s, s}(\partial \Omega), 1<s<\infty,
$$

there exists a unique solution $(u, p) \in W^{1, s}(\Omega) \times L^{s}(\Omega)$ ( $p$ is unique up to a constant) satisfying

$$
\begin{cases}\operatorname{div}\left(\sigma_{\mu}(u, p)\right)=f & \text { in } \Omega, \\ \operatorname{div} u=g & \text { in } \Omega, \\ u=\psi & \text { on } \partial \Omega .\end{cases}
$$

Moreover, this solution obeys the estimate

$\|u\|_{W^{1, s}(\Omega)}+\|p\|_{L^{s}(\Omega) / \mathbb{R}} \leq C\left(\|f\|_{W^{-1, s}(\Omega)}+\|g\|_{L^{s}(\Omega)}+\|\psi\|_{W^{1-(1 / s), s}(\partial \Omega)}\right)$,

where $C$ depends on $s, \Omega, \min _{x \in \bar{\Omega}} \mu$ and $\|\mu\|_{C^{1,1}(\bar{\Omega})}$.

Lemma 5 (see [14], Theorem 11). Suppose $\mu \in C^{r, 1}(\bar{\Omega})$ with the integer $r=\max \{m, 1\}, m \geq 0$. For any,

$f \in W^{m, s}(\Omega), g \in W^{m+1, s}(\Omega), \psi \in W^{m+2-(1 / s), s}(\partial \Omega), 1<s<\infty$,

there exists a unique solution $(u, p) \in W^{m+2, s}(\Omega) \times W^{m+1, s}(\Omega)$ ( $p$ is unique up to a constant) satisfying (11). Moreover, this solution obeys the estimate

$\|u\|_{W^{m+2, s}(\Omega)}+\|p\|_{W^{m+1, s}(\Omega) / \mathbb{R}} \leq C\left(\|f\|_{W^{m, s}(\Omega)}+\|g\|_{W^{m+1, s}(\Omega)}+\|\psi\|_{W^{m+2-(1 /), s}(\partial \Omega)}\right)$,

where $C$ depends on $m, s, \Omega$, $\min _{x \in \bar{\Omega}} \mu$, and $\|\mu\|_{C^{r, 1}(\bar{\Omega})}$.
Lemma 6 (see [19], Lemma 1.4, Chapter II). Let $X$ be a finite-dimensional Hilbert space with inner product $[\cdot, \cdot]$ and norm $\|\cdot\|$. And let $P$ be a continuous map from $X$ to itself such that

$$
[P(\zeta), \zeta]>0 \text { for }\|\zeta\|=k>0
$$

Then, there exists $\zeta \in X$ with $\|\zeta\| \leq k$ so that $P(\zeta)=0$.

As presented in the introduction, we assume $\phi_{i}=\varepsilon \psi_{i}(i=$ $1,2)$ with $\psi_{i} \in H^{3 / 2}(\partial \Omega)$ and look for $\left(u^{\varepsilon}, p^{\varepsilon}, B^{\varepsilon}\right)=\left(\varepsilon u_{\varepsilon}, \varepsilon p_{\varepsilon}\right.$, $\left.\varepsilon B_{\varepsilon}\right)$ satisfies (9). Then, the problem (9) is reduced to

$$
\begin{cases}\operatorname{div}\left(\sigma_{\mu}\left(u_{\varepsilon}, p_{\varepsilon}\right)\right)-\varepsilon u_{\varepsilon} \cdot \nabla u_{\varepsilon}+\varepsilon B_{\varepsilon} \cdot \nabla B_{\varepsilon}=0 & \text { in } \Omega, \\ \operatorname{div}\left(\tilde{\sigma}_{\lambda}\left(B_{\varepsilon}\right)\right)-\varepsilon u_{\varepsilon} \cdot \nabla B_{\varepsilon}+\varepsilon B_{\varepsilon} \cdot \nabla u_{\varepsilon}=0 & \text { in } \Omega, \\ \operatorname{div} u_{\varepsilon}=0, \operatorname{div} B_{\varepsilon}=0 & \text { in } \Omega, \\ u_{\varepsilon}=\psi_{1}, B_{\varepsilon}=\psi_{2} & \text { on } \partial \Omega .\end{cases}
$$

We will prove the problem (16) with the solution $u_{\varepsilon}=u_{0}$ $+\varepsilon u, p_{\varepsilon}=p_{0}+\varepsilon p, B_{\varepsilon}=B_{0}+\varepsilon B$, where $\left(u_{0}, p_{0}, B_{0}\right)$ satisfies the Stokes equation

$$
\begin{cases}\operatorname{div}\left(\sigma_{\mu}\left(u_{0}, p_{0}\right)\right)=0 & \text { in } \Omega, \\ \operatorname{div} u_{0}=0 & \text { in } \Omega, \\ u_{0}=\psi_{1} & \text { on } \partial \Omega,\end{cases}
$$

$$
\begin{cases}\operatorname{div}\left(\bar{\sigma}_{\lambda}\left(B_{0}\right)\right)=0 & \text { in } \Omega, \\ \operatorname{div} B_{0}=0 & \text { in } \Omega, \\ B_{0}=\psi_{2} & \text { on } \partial \Omega .\end{cases}
$$

$(u, p, B)$ satisfies

$$
\begin{cases}-\operatorname{div}\left(\sigma_{\mu}(u, p)\right) & +\varepsilon\left(u_{0} \cdot \nabla\right) u+\varepsilon(u \cdot \nabla) u_{0}+\varepsilon^{2}(u \cdot \nabla) u-\varepsilon\left(B_{0} \cdot \nabla\right) B-\varepsilon(B \cdot \nabla) B_{0}-\varepsilon^{2}(B \cdot \nabla) B \\ & =\left(B_{0} \cdot \nabla\right) B_{0}-\left(u_{0} \cdot \nabla\right) u_{0} \triangleq f_{1} \operatorname{in} \Omega, \\ -\operatorname{div}\left(\tilde{\sigma}_{\lambda}(B)\right) & -\varepsilon\left(B_{0} \cdot \nabla\right) u-\varepsilon(B \cdot \nabla) u_{0}-\varepsilon^{2}(B \cdot \nabla) u+\varepsilon(u \cdot \nabla) B_{0}+\varepsilon\left(u_{0} \cdot \nabla\right) B+\varepsilon^{2}(u \cdot \nabla) B \\ & =-\left(u_{0} \cdot \nabla\right) B_{0}+\left(B_{0} \cdot \nabla\right) u_{0} \triangleq f_{2} \operatorname{in} \Omega, \\ \operatorname{div} u=0, & \operatorname{div} B=0 \operatorname{in} \Omega, \\ u=0, & B=0 \text { on } \partial \Omega .\end{cases}
$$


By Lemma 5, we can know that for each $\psi_{1}, \psi_{2} \in H^{3 / 2}$ $(\partial \Omega)$ there exists a unique $\left(u_{0}, p_{0}, B_{0}\right) \in H^{2}(\Omega) \times H^{1}(\Omega) \times$ $H^{2}(\Omega)$ ( $p_{0}$ is unique up to a constant) satisfying (17) and (18) and the estimate

$\left\|u_{0}\right\|_{H^{2}(\Omega)}+\left\|p_{0}\right\|_{H^{1}(\Omega) / \mathbb{R}} \leq C\left\|\psi_{1}\right\|_{H^{3 / 2}(\partial \Omega)} ;\left\|B_{0}\right\|_{H^{2}(\Omega)} \leq C\left\|\psi_{2}\right\|_{H^{3 / 2}(\partial \Omega)}$,

with $\left\|p_{0}\right\|_{H^{1}(\Omega) / \mathbb{R}}:=\inf f_{c \in \mathbb{R}}\left\|p_{0}+c\right\|_{H^{1}(\Omega)}$. In view of the Sobolev imbedding theorems $H^{2}(\Omega) \hookrightarrow C^{0}(\bar{\Omega})$ and $H^{1}(\Omega) \hookrightarrow$ $L^{4}(\Omega)$, we get that

$$
\begin{aligned}
\left\|f_{1}\right\|_{H^{1}(\Omega)} & =\left\|\left(u_{0} \cdot \nabla\right) u_{0}-\left(B_{0} \cdot \nabla\right) B_{0}\right\|_{H^{1}(\Omega)} \\
& \leq C\left(\left\|\psi_{1}\right\|_{H^{3 / 2}(\partial \Omega)}^{2}+\left\|\psi_{2}\right\|_{H^{3 / 2}(\partial \Omega)}^{2}\right), \\
\left\|f_{2}\right\|_{H^{1}(\Omega)} & =\left\|\left(B_{0} \cdot \nabla\right) u_{0}-\left(u_{0} \cdot \nabla\right) B_{0}\right\|_{H^{1}(\Omega)} \\
& \leq C\left(\left\|\psi_{1}\right\|_{H^{3 / 2}(\partial \Omega)}^{2}+\left\|\psi_{2}\right\|_{H^{3 / 2}(\partial \Omega)}^{2}\right) .
\end{aligned}
$$

In order to solve the problem (19), we first prove the existence of (19).

Theorem 7. There exists a positive number $\varepsilon_{0}$ depending on $\psi_{1}, \psi_{2}$ such that for any $|\varepsilon| \leq \varepsilon_{0}$, problem (19) has at least one weak solution $(u, p, B) \in H_{0}^{1}(\Omega) \times L^{2}(\Omega) \times H_{0}^{1}(\Omega)$.

Proof. Similar to Chapter II, Section 1 in [19], we use the Galerkin method to solve the problem (19). We denote $V=\left\{u \in H_{0}^{1}(\Omega) ; \operatorname{div} u=0\right\}, \mathbb{V}=V \times V .\|(u, B)\|_{\mathbb{V}}=$ $\left(\|u\|_{H^{1}(\Omega)}^{2}+\|B\|_{H^{1}(\Omega)}^{2}\right)^{1 / 2}$. By Korn's inequality and Poincare's inequality, it is easily to prove that $H_{0}^{1}(\Omega)$ is a separable Hilbert space with respect to the inner product

$$
<u, w>=\int_{\Omega} S(u) \cdot S(\bar{w}) d x .
$$

Note that $V$ is a closed subspace of $H_{0}^{1}(\Omega)$, which is also separable. Let $\omega_{1}, \omega_{2}, \cdots$ be elements of $\mathscr{V}$ which form a complete orthonormal system of $V$, where

$$
\mathscr{V}:=\left\{w \in C_{0}^{\infty}(\Omega), \operatorname{div} w=0\right\} .
$$

Let $u_{n}=\sum_{j=1}^{n} \xi_{j, n} \omega_{j}, B_{n}=\sum_{j=1}^{n} \zeta_{j, n} \omega_{j}$ with $\xi_{j, n}, \zeta_{j, n} \in \mathbb{C}$ satisfy

$$
\begin{aligned}
& \mu<u_{n}, \omega_{j}>+\varepsilon b\left(u_{0}, u_{n}, \omega_{j}\right)+\varepsilon b\left(u_{n}, u_{0}, \omega_{j}\right)+\varepsilon^{2} b\left(u_{n}, u_{n}, \omega_{j}\right), \\
& -\varepsilon b\left(B_{0}, B_{n}, \omega_{j}\right)-\varepsilon b\left(B_{n}, B_{0}, \omega_{j}\right)-\varepsilon^{2} b\left(B_{n}, B_{n}, \omega_{j}\right)=\left(f_{1}, \omega_{j}\right)
\end{aligned}
$$

$\lambda<B_{n}, \omega_{j}>-\varepsilon b\left(B_{0}, u_{n}, \omega_{j}\right)-\varepsilon b\left(B_{n}, u_{0}, \omega_{j}\right)-\varepsilon^{2} b\left(B_{n}, u_{n}, \omega_{j}\right)$,

$$
+\varepsilon b\left(u_{n}, B_{0}, \omega_{j}\right)+\varepsilon b\left(u_{0}, B_{n}, \omega_{j}\right)+\varepsilon^{2} b\left(u_{n}, B_{n}, \omega_{j}\right)=\left(f_{2}, \omega_{j}\right),
$$

for $j=1,2, \cdots, n$, where

$$
\begin{aligned}
b(u, v, w) & =\int_{\Omega}(u \cdot \nabla) v \cdot \bar{w} d x, \\
(f, w) & =\int_{\Omega} f \cdot \bar{w} d x .
\end{aligned}
$$

Analogous to Lemma 1.3, Chapter II in [19], we can easily prove two properties of $\mathrm{b}(u, v, w)$

$$
\begin{aligned}
& b(u, v, v)=0 \text { for all } u \in V, v \in H_{0}^{1}(\Omega), \\
& b(u, v, w)=-b(u, w, v) \text { for all } u \in V, v, w \in H_{0}^{1}(\Omega) .
\end{aligned}
$$

Moreover, by the imbedding theorem $H^{2}(\Omega)^{\circ} C^{0}(\bar{\Omega})$, we also can get that

$$
\left\{\begin{aligned}
\left|b\left(u, u, u_{0}\right)\right| & =\left|\int_{\Omega}(u \cdot \nabla) u \cdot u_{0} d x\right| \leq C\left\|u_{0}\right\|_{H^{2}(\Omega)}\|u\|_{H^{1}(\Omega)}^{2}, \\
\left|b\left(B, B, B_{0}\right)\right| & =\left|\int_{\Omega}(B \cdot \nabla) B \cdot B_{0} d x\right| \leq C\left\|B_{0}\right\|_{H^{2}(\Omega)}\|B\|_{H^{1}(\Omega)}^{2}, \\
\left|b\left(u_{0}, u, u\right)\right| & =\left|\int_{\Omega}\left(u_{0} \cdot \nabla\right) u \cdot u d x\right| \leq C\left\|u_{0}\right\|_{H^{2}(\Omega)}\|u\|_{H^{1}(\Omega)}^{2}, \\
\left|b\left(B_{0}, B, B\right)\right| & =\left|\int_{\Omega}\left(B_{0} \cdot \nabla\right) B \cdot B d x\right| \leq C\left\|B_{0}\right\|_{H^{2}(\Omega)}\|B\|_{H^{1}(\Omega)}^{2},
\end{aligned}\right.
$$

with $u_{0}, B_{0} \in H^{2}(\Omega)$.

Let $A=$ the space by $\omega_{1}, \omega_{2}, \cdots, \omega_{n}$ and the inner product $[\cdot, \cdot]_{A}$ is inducted by that of $V$, namely, $<\cdot, \cdot>$ given in (23). We choose $X=A \times A$, and the inner product $[\cdot, \cdot]_{X}$ is defined by

$$
\begin{aligned}
{[(u, B),(v, D)]_{X} } & =[u, v]_{A}+[B, D]_{A} \\
& =\langle u, v>+<B, D>, \forall(u, B) \in X,(v, D) \in X,
\end{aligned}
$$

where the norm $\|(u, B)\|_{X}=\left(\|u\|_{H^{1}(\Omega)}^{2}+\|B\|_{H^{1}(\Omega)}^{2}\right)^{1 / 2}$. We define $P=P_{n}: X=A \times A \longrightarrow X=A \times A$ by

$$
\begin{aligned}
& {\left[P_{n}(u, B),(v, D)\right]_{X} } \\
&= \mu<u, v>+\varepsilon b\left(u_{0}, u, v\right)+\varepsilon b\left(u, u_{0}, v\right)+\varepsilon^{2} b(u, u, v) \\
&-\varepsilon b\left(B_{0}, B, v\right)-\varepsilon b\left(B, B_{0}, v\right)-\varepsilon^{2} b(B, B, v)-\left(f_{1}, v\right) \\
&-\lambda<B, D>+\varepsilon b\left(B_{0}, u, D\right)+\varepsilon b\left(B, u_{0}, D\right)+\varepsilon^{2} b(B, u, D) \\
&-\varepsilon b\left(u, B_{0}, D\right)-\varepsilon b\left(u_{0}, B, D\right)-\varepsilon^{2} b(u, B, D)+\left(f_{2}, D\right),
\end{aligned}
$$


for any $(u, B),(v, D) \in X$. The continuously of $P_{n}$ is obvious. To verify (26) and (28), we can see with the help with (30), (31), and (32) that

$$
\begin{aligned}
& {\left[P_{n}(u, B),(u, B)\right]_{X}} \\
& =\mu\left\langle u, u>+\varepsilon b\left(u_{0}, u, u\right)+\varepsilon b\left(u, u_{0}, u\right)+\varepsilon^{2} b(u, u, u)\right. \\
& -\varepsilon b\left(B_{0}, B, u\right)-\varepsilon b\left(B, B_{0}, u\right)-\varepsilon^{2} b(B, B, u)-\left(f_{1}, u\right) \\
& -\lambda<B, B>-\varepsilon b\left(B_{0}, u, B\right)-\varepsilon b\left(B, u_{0}, B\right)-\varepsilon^{2} b(B, u, B) \\
& +\varepsilon b\left(u, B_{0}, B\right)+\varepsilon b\left(u_{0}, B, B\right)+\varepsilon^{2} b(u, B, B)+\left(f_{2}, B\right) \\
& \geq C\|(u, B)\|_{X}^{2}-\left|\varepsilon b\left(u, u, u_{0}\right)\right|-\left|\varepsilon b\left(B_{0}, B, u\right)\right|-\left|\varepsilon b\left(B, B_{0}, u\right)\right|-\left|\left(f_{1} u\right)\right| \\
& -\left|\varepsilon b\left(B_{0}, u, B\right)\right|-\left|\varepsilon b\left(B, u_{0}, B\right)\right|-\left|\left(f_{2}, B\right)\right| \\
& \geq C\|(u, B)\|_{X}^{2}-\varepsilon C^{\prime}\left\|u_{0}\right\|_{H^{2}}\|u\|_{H^{1}}^{2}-\varepsilon C^{\prime}\left\|B_{0}\right\|_{H^{2}}\|B\|_{H^{1}}\|u\|_{H^{1}}-\left\|f_{1}\right\|_{H^{-1}}\|u\|_{H^{1}} \\
& -\varepsilon C^{\prime}\left\|B_{0}\right\|_{H^{2}}\|B\|_{H^{1}}\|u\|_{H^{1}}-\varepsilon C^{\prime}\left\|u_{0}\right\|_{H^{2}}\|B\|_{H^{1}}^{2}-\left\|f_{2}\right\|_{H^{-1}}\|B\|_{H^{1}} \\
& \geq C\|(u, B)\|_{X}^{2}-\varepsilon C^{\prime}\left\|u_{0}\right\|_{H^{2}}\|(u, B)\|_{X}^{2}-\varepsilon C^{\prime}\left\|B_{0}\right\|_{H^{2}}\|(u, B)\|_{X}^{2}-\left\|f_{1}\right\|_{H^{-1}}\|(u, B)\|_{X^{-}}\left\|f_{2}\right\|_{H^{-1}}\|(u, B)\|_{X} \\
& \geq\|(u, B)\|_{X}\left\{\left(C-\varepsilon C^{\prime}\left\|u_{0}\right\|_{H^{2}}-\varepsilon C^{\prime}\left\|B_{0}\right\|_{H^{2}}\right)\|(u, B)\|_{X}-\left\|f_{1}\right\|_{H^{-1}}-\left\|f_{2}\right\|_{H^{-1}}\right\} \text {, }
\end{aligned}
$$

where $C$ and $C^{\prime}$ are positive numbers. Therefore, if we choose a small $\varepsilon_{0}$, depending on $\psi_{1}, \psi_{2}$, such that

$$
C-\varepsilon C^{\prime}\left\|u_{0}\right\|_{H^{2}}-\varepsilon C^{\prime}\left\|B_{0}\right\|_{H^{2}}>0, \forall|\varepsilon| \leq \varepsilon_{0},
$$

then $\left[P_{n}(u, B),(u, B)\right]>0$ for $\|(u, B)\|_{X}=k$ with

$$
k>\frac{\left\|f_{1}\right\|_{H^{-1}}+\left\|f_{2}\right\|_{H^{-1}}}{C-\varepsilon C^{\prime}\left\|u_{0}\right\|_{H^{2}}-\varepsilon C^{\prime}\left\|B_{0}\right\|_{H^{2}}} \text { for }|\varepsilon| \leq \varepsilon_{0} .
$$

Hence, by Lemma (15), we can obtain the existence of $u_{n}$, $B_{n}$ satisfying (26) and (28). Now, we would like to pass the limit of $u_{n}, B_{n}$. Multiplying (26) by $\bar{\xi}_{j, n}$ and summing the corresponding equalities from 1 and $n$, we can get

$$
\begin{aligned}
\mu & <u_{n}, u_{n}>+\varepsilon b\left(u_{0}, u_{n}, u_{n}\right)+\varepsilon b\left(u_{n}, u_{0}, u_{n}\right)+\varepsilon^{2} b\left(u_{n}, u_{n}, u_{n}\right) \\
& -\varepsilon b\left(B_{0}, B_{n}, u_{n}\right)-\varepsilon b\left(B_{n}, B_{0}, u_{n}\right)-\varepsilon^{2} b\left(B_{0}, B_{n}, u_{n}\right)=\left(f_{1}, u_{n}\right) .
\end{aligned}
$$

Similarly, multiplying (28) by $\bar{\zeta}_{j, n}$ and summing the corresponding equalities from 1 and $n$ gives

$$
\lambda<B_{n}, B_{n}>-\varepsilon b\left(B_{0}, u_{n}, B_{n}\right)-\varepsilon b\left(B_{n}, u_{0}, B_{n}\right)-\varepsilon^{2} b\left(B_{n}, u_{n}, B_{n}\right)
$$

$+\varepsilon b\left(u_{n}, B_{0}, B_{n}\right)+\varepsilon b\left(u_{0}, B_{n}, B_{n}\right)+\varepsilon^{2} b\left(u_{n}, B_{n}, B_{n}\right)=\left(f_{2}, B_{n}\right)$.
Using (30)-(40), we can get that

$$
\left\|\left(u_{n}, B_{n}\right)\right\|_{\mathbb{V}} \leq C_{0}\left(\left\|f_{1}\right\|_{H^{-1}(\Omega)}+\left\|f_{2}\right\|_{H^{-1}(\Omega)}\right),
$$

where $C_{0}>0$ is uniforming in $\varepsilon$ provided $|\varepsilon| \leq \varepsilon_{0}$. Therefore, there exists $(u, B)$ in $\mathbb{V}$ and two subsequences $\left\{u_{n^{\prime}}\right\},\left\{B_{n^{\prime}}\right\}$ such that

$$
u_{n^{\prime}} \rightarrow u \text { weakly in } V \text { as } n^{\prime} \longrightarrow \infty \text {, }
$$

$$
B_{n^{\prime}} \rightarrow B \text { weakly in } V \text { as } n^{\prime} \longrightarrow \infty \text {. }
$$

By the Sobolev imbedding theorem, we have that

$$
u_{n^{\prime}} \longrightarrow u \text { strongly in } L^{4}(\Omega) \text { as } n^{\prime} \longrightarrow \infty
$$

$$
B_{n^{\prime}} \longrightarrow B \text { strongly in } L^{4}(\Omega) \text { as } n^{\prime} \longrightarrow \infty \text {. }
$$

By using (42)-(45), for any $\omega \in \mathscr{V}$, we can obtain that

$$
\begin{aligned}
& \left|b\left(u_{n}{ }^{\prime}, u_{n}{ }^{\prime}, \omega\right)-b(u, u, \omega)\right| \\
& \quad \leq\left|\int_{\Omega}\left(u_{n}{ }^{\prime} \cdot \nabla\right) \omega \cdot\left(u_{n}{ }^{\prime}-u\right) d x\right|+\left|\int_{\Omega}\left(\left(u_{n}{ }^{\prime}-u\right) \cdot \nabla\right) \omega \cdot-u d x\right| \\
& \quad \leq\left\|u_{n}^{\prime}\right\|_{L^{4}}\|\nabla \omega\|_{L^{2}}\left\|u_{n}{ }^{\prime}-u\right\|_{L^{4}+\left\|u_{n}{ }^{\prime}-u\right\|_{L^{4}}\|\nabla \omega\|_{L^{2}}\|u\|_{L^{4}}} \\
& \quad \longrightarrow 0\left(\operatorname{as~} n^{\prime} \longrightarrow \infty\right) .
\end{aligned}
$$


Similarly, when $n^{\prime} \longrightarrow \infty$ we can get that

$b\left(u_{0}, u_{n}{ }^{\prime}, \omega\right) \longrightarrow b\left(u_{0}, u, \omega\right), b\left(u_{n}{ }^{\prime}, u_{0}, \omega\right) \longrightarrow b\left(u, u_{0}, \omega\right)$,

$b\left(B_{0}, B_{n}{ }^{\prime}, \omega\right) \longrightarrow b\left(B_{0}, B, \omega\right), b\left(B_{n}{ }^{\prime}, B_{0}, \omega\right) \longrightarrow b\left(B, B_{0}, \omega\right)$,

$b\left(B_{n}{ }^{\prime}, B_{n}{ }^{\prime}, \omega\right) \longrightarrow b(B, B, \omega), b\left(B_{0}, u_{n}{ }^{\prime}, \omega\right) \longrightarrow b\left(B_{0}, u, \omega\right)$,

$b\left(B_{n}{ }^{\prime}, u_{0}, \omega\right) \longrightarrow b\left(B, u_{0}, \omega\right), b\left(B_{n}{ }^{\prime}, u_{n}{ }^{\prime}, \omega\right) \longrightarrow b(B, u, \omega)$,

$b\left(u_{n}{ }^{\prime}, B_{0}, \omega\right) \longrightarrow b\left(u, B_{0}, \omega\right), b\left(u_{0}, B_{n}{ }^{\prime}, \omega\right) \longrightarrow b\left(u_{0}, B, \omega\right)$,

$b\left(u_{n}{ }^{\prime}, B_{n}{ }^{\prime}, \omega\right) \longrightarrow b(u, B, \omega)$.

with $\omega \in \mathscr{V}$.

Therefore, limiting $u_{n}{ }^{\prime}, B_{n}{ }^{\prime}$ in (26) and (28), we show that

$$
\left\{\begin{aligned}
\mu<u, \omega_{j}>\quad & +\varepsilon b\left(u_{0}, u, \omega_{j}\right)+\varepsilon b\left(u, u_{0}, \omega_{j}\right)+\varepsilon^{2} b\left(u, u, \omega_{j}\right) \\
& -\varepsilon b\left(B_{0}, B, \omega_{j}\right)-\varepsilon b\left(B, B_{0}, \omega_{j}\right)-\varepsilon^{2} b\left(B, B, \omega_{j}\right)=\left(f_{1}, \omega_{j}\right), \\
\lambda<B, \omega_{j}>\quad & -\varepsilon b\left(B_{0}, u, \omega_{j}\right)-\varepsilon b\left(B, u_{0}, \omega_{j}\right)-\varepsilon^{2} b\left(B, u, \omega_{j}\right) \\
& +\varepsilon b\left(u, B_{0}, \omega_{j}\right)+\varepsilon b\left(u_{0}, B, \omega_{j}\right)+\varepsilon^{2} b\left(u, B, \omega_{j}\right)=\left(f_{2}, \omega_{j}\right),
\end{aligned}\right.
$$

for $j=1,2, \cdots$. Since $\omega_{1}, \omega_{2}, \cdots$ is a complete orthonormal system of $V$, we can obtain that

$$
\left\{\begin{aligned}
\mu<u, \omega\rangle & +\varepsilon b\left(u_{0}, u, \omega\right)+\varepsilon b\left(u, u_{0}, \omega\right)+\varepsilon^{2} b(u, u, \omega) \\
& -\varepsilon b\left(B_{0}, B, \omega\right)-\varepsilon b\left(B, B_{0}, \omega\right)-\varepsilon^{2} b(B, B, \omega)=\left(f_{1}, \omega\right), \\
\lambda\langle B, \omega\rangle \quad & -\varepsilon b\left(B_{0}, u, \omega\right)-\varepsilon b\left(B, u_{0}, \omega\right)-\varepsilon^{2} b(B, u, \omega) \\
& +\varepsilon b\left(u, B_{0}, \omega\right)+\varepsilon b\left(u_{0}, B, \omega\right)+\varepsilon^{2} b(u, B, \omega)=\left(f_{2}, \omega\right),
\end{aligned}\right.
$$

for all $\omega \in V$. Thus, there exists $p \in L^{2}(\Omega)$ (and $u, B \in V$ ) such that

$$
\begin{cases}-\operatorname{div}\left(\sigma_{\mu}(u, p)\right) & +\varepsilon\left(u_{0} \cdot \nabla\right) u+\varepsilon(u \cdot \nabla) u_{0}+\varepsilon^{2}(u \cdot \nabla) u \\ & -\varepsilon\left(B_{0} \cdot \nabla\right) B-\varepsilon(B \cdot \nabla) B_{0}-\varepsilon^{2}(B \cdot \nabla) B=f_{1}, \\ -\operatorname{div}\left(\tilde{\sigma}_{\lambda}(B)\right) & -\varepsilon\left(B_{0} \cdot \nabla\right) u-\varepsilon(B \cdot \nabla) u_{0}-\varepsilon^{2}(B \cdot \nabla) u \\ & +\varepsilon(u \cdot \nabla) B_{0}+\varepsilon\left(u_{0} \cdot \nabla\right) B+\varepsilon^{2}(u \cdot \nabla) B=f_{2},\end{cases}
$$

in the weak sense.

Now, the existence of weak solution $(u, p, B)$ to (19) is proved. To show the dependence of $(u, p, B)$ on $\varepsilon$, we define $u=\tilde{u}_{\varepsilon}, B=\tilde{B}_{\varepsilon}$, and $p=\tilde{p}_{\varepsilon}$. Next, in order to show that the uniformly boundedness of $\left(\tilde{u}_{\varepsilon}, \tilde{p}_{\varepsilon}, \tilde{B}_{\varepsilon}\right)$ about $\varepsilon$ in some Sobolev space, we will discuss the regularity of $\left(\tilde{u}_{\varepsilon}, \tilde{p}_{\varepsilon}, \tilde{B}_{\varepsilon}\right)$. This uniformly boundedness of $\left(\tilde{u}_{\varepsilon}, \tilde{p}_{\varepsilon}, \tilde{B}_{\varepsilon}\right)$ makes us to consider the limiting behavior of $\left(\varepsilon^{-1} u_{\varepsilon}, \varepsilon^{-1} p_{\varepsilon}, \varepsilon^{-1} B_{\varepsilon}\right)$. The proof of regu- larity for $\left(\tilde{u}_{\varepsilon}, \tilde{p}_{\varepsilon}, \tilde{B}_{\varepsilon}\right)$ relies on the regularity result for the Stokes equations and the "bootstrapping" technique. Some arguments used here are derived from [19].

Theorem 8. Let $\left(\tilde{u}_{\varepsilon}, \tilde{p}_{\varepsilon}, \tilde{B}_{\varepsilon}\right)$ be a weak solution of (19) for $|\varepsilon| \leq \varepsilon_{0}$. Assume $\varepsilon_{0}<1$, then $\left(\tilde{u}_{\varepsilon}, \tilde{p}_{\varepsilon}, \tilde{B}_{\varepsilon}\right) \in H^{2}(\Omega) \times H^{1}(\Omega)$ $\times H^{2}(\Omega)$ and satisfies

$\left\|\tilde{\mathcal{u}}_{\varepsilon}\right\|_{H^{2}(\Omega)}+\left\|\tilde{p}_{\varepsilon}\right\|_{H^{1}(\Omega) / R}+\left\|\tilde{B}_{\varepsilon}\right\|_{H^{2}(\Omega)} \leq \Sigma_{j=1}^{4}\left(\left\|\psi_{1}\right\|_{H^{3 / 2}(\partial \Omega)}^{j^{3}}+\left\|\psi_{2}\right\|_{H^{3 / 2}(\partial \Omega)}^{2^{j}}\right)$.

Proof. For conveniently, we denote $u=\tilde{u}_{\varepsilon}, p=\tilde{p}_{\varepsilon}$, and $B=\tilde{B}_{\varepsilon}$. We now write the first equation and the second equation in the form of Stokes equation

$$
\begin{gathered}
\operatorname{div}\left(\sigma_{\mu}(u, p)\right)=g_{1}, \operatorname{div} u=0, \\
\operatorname{div}\left(\tilde{\sigma}_{\lambda}(B)\right)=g_{2}, \operatorname{div} B=0,
\end{gathered}
$$

with

$$
\begin{aligned}
g_{1}= & \varepsilon\left(u_{0} \cdot \nabla\right) u+\varepsilon(u \cdot \nabla) u_{0}+\varepsilon^{2}(u \cdot \nabla) u \\
& -\varepsilon\left(B_{0} \cdot \nabla\right) B-\varepsilon(B \cdot \nabla) B_{0}-\varepsilon^{2}(B \cdot \nabla) B-f_{1}, \\
g_{2}= & \varepsilon\left(B_{0} \cdot \nabla\right) u+\varepsilon(B \cdot \nabla) u_{0}+\varepsilon^{2}(B \cdot \nabla) u \\
& -\varepsilon(u \cdot \nabla) B_{0}-\varepsilon\left(u_{0} \cdot \nabla\right) B-\varepsilon^{2}(u \cdot \nabla) B-f_{2} .
\end{aligned}
$$

From the proof of the existence, we have that $u$, $B \in H_{0}^{1}(\Omega)$ and

$$
\begin{aligned}
& \|u\|_{H^{1}(\Omega)} \leq C\left(\left\|f_{1}\right\|_{H^{-1}(\Omega)}+\left\|f_{2}\right\|_{H^{-1}(\Omega)}\right) \leq C\left(\left\|\psi_{1}\right\|_{H^{3 / 2}(\partial \Omega)}^{2}+\left\|\psi_{2}\right\|_{H^{3 / 2}(\partial \Omega)}^{2}\right), \\
& \|B\|_{H^{1}(\Omega)} \leq C\left(\left\|f_{1}\right\|_{H^{-1}(\Omega)}+\left\|f_{2}\right\|_{H^{-1}(\Omega)}\right) \leq C\left(\left\|\psi_{1}\right\|_{H^{3 / 2}(\partial \Omega)}^{2}+\left\|\psi_{2}\right\|_{H^{3 / 2}(\partial \Omega)}^{2}\right) .
\end{aligned}
$$

By the Sobolev imbedding, we can get $u, B \in L^{6}(\Omega)$. Subsequently, we obtain that $(u \cdot \nabla) u \in L^{3 / 2}(\Omega),(B \cdot \nabla)$ $B \in L^{3 / 2}(\Omega)$ and

$$
\begin{aligned}
\|(u \cdot \nabla) u\|_{L^{3 / 2}(\Omega)} & \leq C\|u\|_{L^{6}(\Omega)}\|\nabla u\|_{L^{2}(\Omega)} \leq C\|u\|_{H^{1}(\Omega)}^{2} \\
& \leq C\left(\left\|\psi_{1}\right\|_{H^{3 / 2}(\partial \Omega)}^{4}+\left\|\psi_{2}\right\|_{H^{3 / 2}(\partial \Omega)}^{4}\right), \\
\|(B \cdot \nabla) B\|_{L^{3 / 2}(\Omega)} & \leq C\|B\|_{L^{6}(\Omega)}\|\nabla B\|_{L^{2}(\Omega)} \leq C\|B\|_{H^{1}(\Omega)}^{2} \\
& \leq C\left(\left\|\psi_{1}\right\|_{H^{3 / 2}(\partial \Omega)}^{4}+\left\|\psi_{2}\right\|_{H^{3 / 2}(\partial \Omega)}^{4}\right) .
\end{aligned}
$$


Similarly, we can get

$\left\|\left(u_{0} \cdot \nabla\right) u\right\|_{L^{3 / 2}(\Omega)} \leq C\left\|u_{0}\right\|_{L^{6}(\Omega)}\|\nabla u\|_{L^{2}(\Omega)} \leq C\left\|u_{0}\right\|_{H^{1}(\Omega)}\|u\|_{H^{1}(\Omega)} \leq C\left\|u_{0}\right\|_{H^{2}(\Omega)}\|u\|_{H^{1}(\Omega)}$ $\leq C\left(\left\|\psi_{1}\right\|_{H^{3 / 2}(\partial \Omega)}^{2}+\left\|\psi_{1}\right\|_{H^{3 / 2}(\partial \Omega)}^{4}+\left\|\psi_{2}\right\|_{H^{3 / 2}(\partial \Omega)}^{4}\right)$,

$\left\|\left(B_{0} \cdot \nabla\right) B\right\|_{L^{3 / 2}(\Omega)} \leq C\left\|B_{0}\right\|_{L^{6}(\Omega)}\|\nabla B\|_{L^{2}(\Omega)} \leq C\left\|B_{0}\right\|_{H^{1}(\Omega)}\|B\|_{H^{1}(\Omega)} \leq C\left\|B_{0}\right\|_{H^{2}(\Omega)}\|B\|_{H^{1}(\Omega)}$ $\leq C\left(\left\|\psi_{2}\right\|_{H^{3 / 2}(\partial \Omega)}^{2}+\left\|\psi_{1}\right\|_{H^{3 / 2}(\partial \Omega)}^{4}+\left\|\psi_{2}\right\|_{H^{3 / 2}(\partial \Omega)}^{4}\right)$,

$\begin{cases}\left\|(u \cdot \nabla) u_{0}\right\|_{L^{3 / 2}(\Omega)} & \leq C\left(\left\|\psi_{1}\right\|_{H^{3 / 2}(\partial \Omega)}^{2}+\left\|\psi_{1}\right\|_{H^{3 / 2}(\partial \Omega)}^{4}+\left\|\psi_{2}\right\|_{H^{3 / 2}(\partial \Omega)}^{4}\right), \\ \left\|(B \cdot \nabla) B_{0}\right\|_{L^{3 / 2}(\Omega)} & \leq C\left(\left\|\psi_{2}\right\|_{H^{3 / 2}(\partial \Omega)}^{2}+\left\|\psi_{1}\right\|_{H^{3 / 2}(\partial \Omega)}^{4}+\left\|\psi_{2}\right\|_{H^{3 / 2}(\partial \Omega)}^{4}\right), \\ \left\|f_{1}\right\|_{L^{3 / 2}(\Omega)} & \leq C\left(\left\|\psi_{1}\right\|_{H^{3 / 2}(\partial \Omega)}^{2}+\left\|\psi_{2}\right\|_{H^{3 / 2}(\partial \Omega)}^{2}\right) .\end{cases}$

Therefore, as $\varepsilon_{0}<1$, we have that $g_{1} \in L^{3 / 2}(\Omega)$ and $\left\|g_{1}\right\|_{L^{3 / 2}(\Omega)} \leq C\left(\left\|\psi_{1}\right\|_{H^{3 / 2}(\partial \Omega)}^{2}+\left\|\psi_{2}\right\|_{H^{3 / 2}(\partial \Omega)}^{2}+\left\|\psi_{1}\right\|_{H^{3 / 2}(\partial \Omega)}^{4}+\left\|\psi_{2}\right\|_{H^{3 / 2}(\partial \Omega)}^{4}\right)$. and

Similarly, from (22) and $\varepsilon_{0}<1$, we have that $g_{2} \in L^{3 / 2}(\Omega)$ $\left\|g_{2}\right\|_{L^{3 / 2}(\Omega)} \leq C\left(\left\|\psi_{1}\right\|_{H^{3 / 2}(\partial \Omega)}^{2}+\left\|\psi_{2}\right\|_{H^{3 / 2}(\partial \Omega)}^{2}+\left\|\psi_{1}\right\|_{H^{3 / 2}(\partial \Omega)}^{4}+\left\|\psi_{2}\right\|_{H^{3 / 2}(\partial \Omega)}^{4}\right)$

The regularity theorem for the Stokes equations (Lemma 5) implies that

$$
\begin{gathered}
\|u\|_{W^{2,3 / 2}(\Omega)}+\|p\|_{W^{1,3 / 2}(\Omega) / R} \leq C\left\|g_{1}\right\|_{L^{3 / 2}(\Omega)} \\
\leq C\left(\left\|\psi_{1}\right\|_{H^{3 / 2}(\partial \Omega)}^{2}+\left\|\psi_{2}\right\|_{H^{3 / 2}(\partial \Omega)}^{2}+\left\|\psi_{1}\right\|_{H^{3 / 2}(\partial \Omega)}^{4}+\left\|\psi_{2}\right\|_{H^{3 / 2}(\partial \Omega)}^{4}\right) \\
\|B\|_{W^{2,3 / 2}(\Omega)} \leq C\left\|g_{2}\right\|_{L^{3 / 2}(\Omega)} \\
\leq C\left(\left\|\psi_{1}\right\|_{H^{3 / 2}(\partial \Omega)}^{2}+\left\|\psi_{2}\right\|_{H^{3 / 2}(\partial \Omega)}^{2}+\left\|\psi_{1}\right\|_{H^{3 / 2}(\partial \Omega)}^{4}+\left\|\psi_{2}\right\|_{H^{3 / 2}(\partial \Omega)}^{4}\right) .
\end{gathered}
$$

The estimates (60) and (62) are not exactly what we want. We need to improve $L^{3 / 2}$ - base Sobolev norms to $L^{2}$ - one on the left-hand side of (60) and (62). This can be achieved by the "bootstrapping" argument. In view of Sobolev imbedding, $W^{2,3 / 2}(\Omega)^{\circ} L^{r}(\Omega)$ for any $1<r<\infty$, we thus obtain that $u \otimes u, B \otimes B \in L^{s}$ for any $s \in(1, \infty)$, and

$$
\begin{aligned}
&\|(u \cdot \nabla) u\|_{W^{-1, s}(\Omega)}=\|\operatorname{div}(u \otimes u)\|_{W^{-1,5}(\Omega)} \\
& \leq C\|u \otimes u\|_{L^{s}(\Omega)} \\
& \leq C\|u\|_{L^{r_{1}}(\Omega)}\|u\|_{L^{r_{2}(\Omega)}} \\
& \leq C\|u\|_{W^{2,3 / 2}(\Omega)}\|u\|_{W^{2,3 / 2}(\Omega)} \\
& \leq C^{\prime \prime}\left(\left\|\psi_{1}\right\|_{H^{\frac{3}{2}}(\partial \Omega)}^{4}+\left\|\psi_{2}\right\|_{H^{\frac{3}{2}}(\partial \Omega)}^{4}+\left\|\psi_{1}\right\|_{H^{\frac{3}{2}}(\partial \Omega)}^{8}+\left\|\psi_{2}\right\|_{H^{\frac{3}{2}}(\partial \Omega)}^{8}\right),
\end{aligned}
$$

where $s^{-1}=r_{1}^{-1}+r_{2}^{-1}$ and $C^{\prime \prime}=C^{\prime \prime}\left(\Omega, r_{1}, r_{2}\right)$. In the proof of (65), we uses the inequality (60) and divu $=0$. Similarly, by (62) and $\operatorname{div} B=0$, we can get

$$
\begin{gathered}
\|(B \cdot \nabla) B\|_{W^{-1, s}(\Omega)} \leq C\|B \otimes B\|_{L^{s}(\Omega)} \leq C\|B\|_{L^{r^{1}}(\Omega)}\|B\|_{L^{r^{2}}(\Omega)} \leq C\|B\|_{W^{2,3 / 2}(\Omega)}^{2} \\
\leq C^{\prime \prime}\left(\left\|\psi_{1}\right\|_{H^{3 / 2}(\partial \Omega)}^{4}+\left\|\psi_{2}\right\|_{H^{3 / 2}(\partial \Omega)}^{4}+\left\|\psi_{1}\right\|_{H^{3 / 2}(\partial \Omega)}^{8}+\left\|\psi_{2}\right\|_{H^{3 / 2}(\partial \Omega)}^{8}\right) .
\end{gathered}
$$

On the other hand, $H^{2}(\Omega)^{\circ} C^{0}(\bar{\Omega}), H^{2}(\Omega)=W^{2,2}(\Omega)^{\circ}$ $W^{2,3 / 2}(\Omega)$. Likewise, we can get that

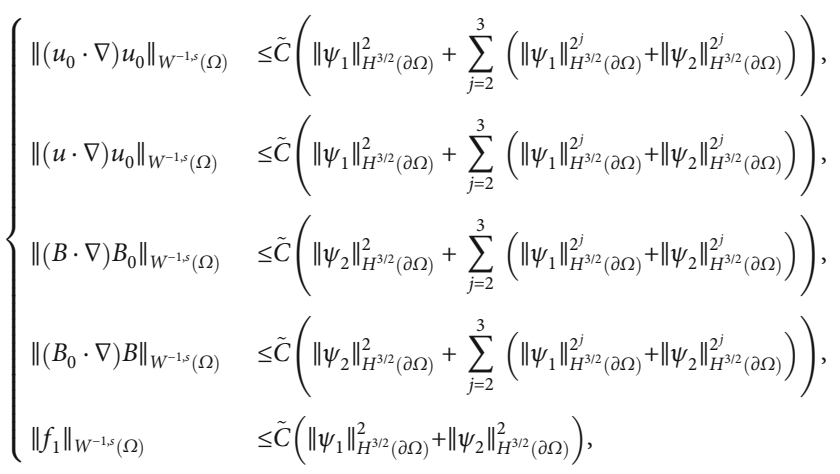

By (65)-(67), we can derive that

$$
\left\|g_{1}\right\|_{W^{-1, s}(\Omega)} \leq C \sum_{j=1}^{3}\left(\left\|\psi_{1}\right\|_{H^{3 / 2}(\partial \Omega)}^{2^{j}}+\left\|\psi_{2}\right\|_{H^{3 / 2}(\partial \Omega)}^{2^{j}}\right) .
$$

Similarly, we can derive that

$$
\left\|g_{2}\right\|_{W^{-1, s}(\Omega)} \leq C \sum_{j=1}^{3}\left(\left\|\psi_{1}\right\|_{H^{3 / 2}(\partial \Omega)}^{2^{j}}+\left\|\psi_{2}\right\|_{H^{3 / 2}(\partial \Omega)}^{2^{j}}\right) .
$$

Therefore, from Lemma 4, we have that

$$
\begin{cases}\|u\|_{W^{1, s}(\Omega)}+\|p\|_{L^{s}(\Omega) / R} & \leq C \sum_{j=1}^{3}\left(\left\|\psi_{1}\right\|_{H^{3 / 2}(\partial \Omega)}^{2^{j}}+\left\|\psi_{2}\right\|_{H^{3 / 2}(\partial \Omega)}^{2^{j}}\right) \\ \|B\|_{W^{1, s}(\Omega)} & \leq C \sum_{j=1}^{3}\left(\left\|\psi_{1}\right\|_{H^{3 / 2}(\partial \Omega)}^{2^{j}}+\left\|\psi_{2}\right\|_{H^{3 / 2}(\partial \Omega)}^{2^{j}}\right)\end{cases}
$$

for any $s \in(1, \infty)$.

Now we consider the estimates of $g_{1}, g_{2}$ in $L^{2}(\Omega)$. From (70), we can get that $(u \cdot \nabla) u,(B \cdot \nabla) B \in L^{s}(\Omega)$ for any $s \in(1, \infty)$ and

$$
\begin{aligned}
\|(u \cdot \nabla) u\|_{L^{s}(\Omega)} & \leq C\|u\|_{L^{r_{1}}(\Omega)}\|\nabla u\|_{L^{r_{2}}(\Omega)} \leq C\|u\|_{W^{1, r_{1}}(\Omega)}\|u\|_{W^{1, r_{2}}(\Omega)} \\
& \leq C \sum_{j=2}^{4}\left(\left\|\psi_{1}\right\|_{H^{3 / 2}(\partial \Omega)}^{j^{j}}+\left\|\psi_{2}\right\|_{H^{3 / 2}(\partial \Omega)}^{2^{j}}\right)
\end{aligned}
$$


with $s^{-1}=r_{1}^{-1}+r_{2}^{-1}$. Similarly, we can get

$$
\|(B \cdot \nabla) B\|_{L^{s}(\Omega)} \leq C \sum_{j=2}^{4}\left(\left\|\psi_{1}\right\|_{H^{3 / 2}(\partial \Omega)}^{2^{j}}+\left\|\psi_{2}\right\|_{H^{3 / 2}(\partial \Omega)}^{2^{j}}\right) .
$$

In particular, we have $(u \cdot \nabla) u,(B \cdot \nabla) B \in L^{2}(\Omega)$ and

$$
\begin{cases}\|(u \cdot \nabla) u\|_{L^{2}(\Omega)} & \leq C \sum_{j=2}^{4}\left(\left\|\psi_{1}\right\|_{H^{3 / 2}(\partial \Omega)}^{2^{j}}+\left\|\psi_{2}\right\|_{H^{3 / 2}(\partial \Omega)}^{2^{j}}\right), \\ \|(B \cdot \nabla) B\|_{L^{2}(\Omega)} & \leq C \sum_{j=2}^{4}\left(\left\|\psi_{1}\right\|_{H^{3 / 2}(\partial \Omega)}^{2^{j}}+\left\|\psi_{2}\right\|_{H^{3 / 2}(\partial \Omega)}^{2^{j}}\right) .\end{cases}
$$

With Sobolev imbedding theorem, we can derive that $\begin{cases}\left\|\left(u_{0} \cdot \nabla\right) u\right\|_{L^{2}(\Omega)} & \leq C\left(\sum_{j=2}^{4}\left(\left\|\psi_{1}\right\|_{H^{3 / 2}(\partial \Omega)}^{2^{j}}+\left\|\psi_{2}\right\|_{H^{3 / 2}(\partial \Omega)}^{2^{j}}\right)+\left\|\psi_{1}\right\|_{H^{3 / 2}(\partial \Omega)}^{2}\right) \\ \left\|(u \cdot \nabla) u_{0}\right\|_{L^{2}(\Omega)} & \leq C\left(\sum_{j=2}^{4}\left(\left\|\psi_{1}\right\|_{H^{3 / 2}(\partial \Omega)}^{j}+\left\|\psi_{2}\right\|_{H^{3 / 2}(\partial \Omega)}^{j}\right)+\left\|\psi_{1}\right\|_{H^{3 / 2}(\partial \Omega)}^{2}\right) \\ \left\|\left(B_{0} \cdot \nabla\right) B\right\|_{L^{2}(\Omega)} & \leq C\left(\sum_{j=2}^{4}\left(\left\|\psi_{1}\right\|_{H^{3 / 2}(\partial \Omega)}^{2^{j}}+\left\|\psi_{2}\right\|_{H^{3 / 2}(\partial \Omega)}^{2^{j}}\right)+\left\|\psi_{2}\right\|_{H^{3 / 2}(\partial \Omega)}^{2}\right) \\ \left\|(B \cdot \nabla) B_{0}\right\|_{L^{2}(\Omega)} & \leq C\left(\sum_{j=2}^{4}\left(\left\|\psi_{1}\right\|_{H^{3 / 2}(\partial \Omega)}^{2^{j}}+\left\|\psi_{2}\right\|_{H^{3 / 2}(\partial \Omega)}^{2^{j}}\right)+\left\|\psi_{2}\right\|_{H^{3 / 2}(\partial \Omega)}^{2}\right) \\ \left\|f_{1}\right\|_{L^{2}(\Omega)} & \leq C\left\|f_{1}\right\|_{H^{1}(\Omega)} \leq C\left(\left\|\psi_{1}\right\|_{H^{3 / 2}(\partial \Omega)}^{2}+\left\|\psi_{2}\right\|_{H^{3 / 2}(\partial \Omega)}^{2}\right) .\end{cases}$

Then, from (73) and (74), we can get

$$
\left\|g_{1}\right\|_{L^{2}(\Omega)} \leq C \sum_{j=1}^{4}\left(\left\|\psi_{1}\right\|_{H^{3 / 2}(\partial \Omega)}^{2^{j}}+\left\|\psi_{2}\right\|_{H^{3 / 2}(\partial \Omega)}^{2^{j}}\right)
$$

Similarly, we have

$$
\left\|g_{2}\right\|_{L^{2}(\Omega)} \leq C \sum_{j=1}^{4}\left(\left\|\psi_{1}\right\|_{H^{3 / 2}(\partial \Omega)}^{2^{j}}+\left\|\psi_{2}\right\|_{H^{3 / 2}(\partial \Omega)}^{2^{j}}\right)
$$

Then, from Lemma 5, we have

$$
\|u\|_{H^{2}(\Omega)}+\|p\|_{H^{1}(\Omega) / R} \leq C \sum_{j=1}^{4}\left(\left\|\psi_{1}\right\|_{H^{3 / 2}(\partial \Omega)}^{2^{j}}+\left\|\psi_{2}\right\|_{H^{3 / 2}(\partial \Omega)}^{2^{j}}\right)
$$

$$
\|B\|_{H^{2}(\Omega)} \leq C \sum_{j=1}^{4}\left(\left\|\psi_{1}\right\|_{H^{3 / 2}(\partial \Omega)}^{2^{j}}+\left\|\psi_{2}\right\|_{H^{3 / 2}(\partial \Omega)}^{2^{j}}\right) .
$$

From inequalities (77) and (78), we can get the inequality (51). The proof of Theorem 8 is completed.

\section{Inverse Problem}

In Section 2,we have proved the existence of the solution $(u, p, B)$ to the system (1) with the boundary data $\left(\varepsilon \psi_{1}, \varepsilon \psi_{2}\right)$ - In this section, we derived the asymptotic behavior of solutions as $\varepsilon \longrightarrow 0$ and prove the theorem in (1)-(5) by linearization technique.

Given any $\psi_{1}, \psi_{2} \in H^{3 / 2}(\partial \Omega)$, let $\left(u_{0}, p_{0}, B_{0}\right) \in H^{2}(\Omega) \times$ $H^{1}(\Omega) \times H^{2}(\Omega)$ be the unique solution $\left(p_{0}\right.$ is unique up to a constant) of two Stokes equations

$$
\begin{aligned}
& \begin{cases}\operatorname{div}\left(\sigma_{\mu}\left(u_{0}, p_{0}\right)\right)=0 & \text { in } \Omega, \\
\operatorname{div} u_{0}=0 & \text { in } \Omega ;\end{cases} \\
& \begin{cases}\operatorname{div}\left(\tilde{\sigma}_{\lambda}\left(B_{0}\right)\right)=0 & \text { in } \Omega, \\
\operatorname{div} B_{0}=0 & \text { in } \Omega .\end{cases}
\end{aligned}
$$

with boundary data $\left.\left(u_{0}, B_{0}\right)\right|_{\partial \Omega}=\left(\psi_{1}, \psi_{2}\right)$. As proved in the previous section, there exists $\left(\mathcal{u}^{\varepsilon}, p^{\varepsilon}, B^{\varepsilon}\right)$ with the form

$$
u^{\varepsilon}=\varepsilon u_{0}+\varepsilon^{2} \tilde{u}_{\varepsilon}, p^{\varepsilon}=\varepsilon p_{0}+\varepsilon^{2} \tilde{p}_{\varepsilon}, B^{\varepsilon}=\varepsilon B_{0}+\varepsilon^{2} \tilde{B}_{\varepsilon},
$$

satisfying (1) with boundary data $\left.\left(u^{\varepsilon}, B^{\varepsilon}\right)\right|_{\partial \Omega}=\left(\varepsilon \psi_{1}, \varepsilon \psi_{2}\right)$ for all $|\varepsilon|<\varepsilon_{0}$, where $\varepsilon_{0}$ depends on $\left\|\psi_{1}\right\|_{H^{3 / 2}(\partial \Omega)}$ and $\left\|\psi_{2}\right\|_{H^{3 / 2}(\partial \Omega)}$. Moreover, $\left(u^{\varepsilon}, p^{\varepsilon}, B^{\varepsilon}\right)$ statisfies (51). We immediately see that

$$
\begin{aligned}
& \left\|\varepsilon^{-1} u^{\varepsilon}-u_{0}\right\|_{H^{2}(\Omega)}=\left\|\varepsilon \tilde{u}_{\varepsilon}\right\|_{H^{2}(\Omega)} \longrightarrow 0, \\
& \left\|\varepsilon^{-1} p^{\varepsilon}-p_{0}\right\|_{H^{1}(\Omega) / R}=\left\|\varepsilon \tilde{p}_{\varepsilon}\right\|_{H^{1}(\Omega) / R} \longrightarrow 0, \\
& \left\|\varepsilon^{-1} B^{\varepsilon}-B_{0}\right\|_{H^{2}(\Omega)}=\left\|\varepsilon \tilde{B}_{\varepsilon}\right\|_{H^{2}(\Omega)} \longrightarrow 0
\end{aligned}
$$

as $\varepsilon \longrightarrow 0$ and therefore

$$
\begin{gathered}
\left\|\left.\varepsilon^{-1} u^{\varepsilon}\right|_{\partial \Omega}-\left.u_{0}\right|_{\partial \Omega}\right\|_{H^{3 / 2}(\partial \Omega)} \longrightarrow 0, \\
\left\|\left.\varepsilon^{-1} B^{\varepsilon}\right|_{\partial \Omega}-\left.B_{0}\right|_{\partial \Omega}\right\|_{H^{3 / 2}(\partial \Omega)} \longrightarrow 0 \\
\left\|\left.\varepsilon^{-1} \sigma_{\mu}\left(u^{\varepsilon}, p^{\varepsilon}\right) \mathbf{n}\right|_{\partial \Omega}-\left.\sigma_{\mu}\left(u_{0}, p_{0}\right) \mathbf{n}\right|_{\partial \Omega}\right\|_{H^{1 / 2}(\partial \Omega)} \longrightarrow 0, \\
\left\|\left.\varepsilon^{-1} \tilde{\sigma}_{\lambda}\left(B^{\varepsilon}\right) \mathbf{n}\right|_{\partial \Omega}-\left.\tilde{\sigma}_{\lambda}\left(B_{0}\right) \mathbf{n}\right|_{\partial \Omega}\right\|_{H^{1 / 2}(\partial \Omega)}
\end{gathered}
$$

provided

$$
\int_{\Omega} p^{\varepsilon} d x=\int_{\Omega} p_{0} d x=0
$$

As in [9], we define the Cauchy data associated to (79) and (80)

$$
\begin{aligned}
& C_{\mu}=\left\{\left(\left.u_{0}\right|_{\partial \Omega},\left.\sigma_{\mu}\left(u_{0}, p_{0}\right) \mathbf{n}\right|_{\partial \Omega}\right)\right\} \subset H^{3 / 2}(\partial \Omega) \times H^{1 / 2}(\partial \Omega), \\
& C_{\lambda}=\left\{\left(\left.B_{0}\right|_{\partial \Omega},\left.\tilde{\sigma}_{\lambda}\left(B_{0}\right) \mathbf{n}\right|_{\partial \Omega}\right)\right\} \subset H^{3 / 2}(\partial \Omega) \times H^{1 / 2}(\partial \Omega),
\end{aligned}
$$

where $\left(u_{0}, p_{0}\right)$ satisfies (79) and $B_{0}$ satisfies (80). Now, the limits (83)-(86) lead to the following result. 
Theorem 9. The Cauchy data $C_{\mu}, C_{\lambda}$ of Stokes equations can be uniquely determined from the Cauchy data $\tilde{C}_{\mu, \lambda}$ of the MHD equations.

That is to say, $C_{\mu_{1}}=C_{\mu_{2}}$ and $C_{\lambda_{1}}=C_{\lambda_{2}}$ if $\tilde{C}_{\mu_{1}, \lambda_{1}}=\tilde{C}_{\mu_{2}, \lambda_{2}}$. So, we reduce the uniqueness question of the inverse problem for the MHD equations to that for the Stokes equations. Therefore, Theorems 1 and 2 follow from Theorem 9 and the unique determination of viscosity for the Stokes equation proved in [9].

Theorem 10 (see [9], Theorem 1.1). Assume that $\mu_{1}(x)$ and $\mu_{2}(x)$ are two viscosity functions satisfying $\mu_{1}, \mu_{2} \in C^{n_{0}}(\bar{\Omega})$ for $n_{0} \geq 8$ and

$$
\partial^{\alpha} \mu_{1}(x)=\partial^{\alpha} \mu_{2}(x) \forall x \in \partial \Omega,|\alpha| \leq 1 .
$$

Let $C_{\mu_{1}}$ and $C_{\mu_{2}}$ be the Cauchy data associated with $\mu_{1}$ and $\mu_{2}$, respectively. If $C_{\mu_{1}}=C_{\mu_{2}}$, then $\mu_{1}=\mu_{2}$.

Theorem 11 (see [9], Corollary 1.4). Let $\partial \Omega$ be convex with nonvanishing Gauss curvature. Assume that $\mu_{1}(x)$ and $\mu_{2}(x)$ are two viscosity functions satisfying $\mu_{1}, \mu_{2} \in C^{n_{0}}(\bar{\Omega})$ for $n_{0} \geq 8$. If $C_{\mu_{1}}=C_{\mu_{2}}$, then $\mu_{1}=\mu_{2}$.

The regularity requirement in Theorem 10 is to make sure that Eskin's method [20] works in our case. We do not know whether it is optimal. Also, we define the Cauchy data of the Stokes equations as a subset of $H^{1 / 2}(\partial \Omega) \times H^{-1 / 2}(\partial \Omega)$ in [9], i.e., $C_{\mu} \subset H^{1 / 2}(\partial \Omega) \times H^{-1 / 2}(\partial \Omega)$. Nevertheless, the same proof in [9].

In order to prove Theorem 3, we cite the unique determination of viscosity for two-dimension Stokes equation which is proved in [12].

Theorem 12 (see [12], Theorem 1.1). Let $\Omega$ be a simply connected bounded domain in $\mathbb{R}^{2}$ with smooth boundary. Suppose that $\mu_{1}$ and $\mu_{2}$ are two viscosity functions for Stokes equations, assume that $\mu_{j} \in C^{3} \bar{\Omega}$ and $\mu_{j}>0$ with

$$
\left.\partial^{\alpha} \mu_{1}\right|_{\partial \Omega}=\left.\partial^{\alpha} \mu_{2}\right|_{\partial \Omega} \text { for all }|\alpha| \leq 1 \text {. }
$$

Let $C_{\mu_{1}}$ and $C_{\mu_{2}}$ be the Cauchy data associated with $\mu_{1}$ and $\mu_{2}$, respectively. If $C_{\mu_{1}}=C_{\mu_{2}}$, then $\mu_{1}=\mu_{2}$ in $\Omega$.

Similar to Theorem 9, we can prove that the conclusion of Theorem 9 is still true when $u, B \in \mathbb{R}^{2}$ and $\Omega \in \mathbb{R}^{2}$. Then, we can use Theorem 9 and Theorem 12 to prove Theorem 3 .

\section{Data Availability}

The data used to support the findings of this study are included within the article.

\section{Conflicts of Interest}

The authors declare that they have no conflicts of interest.

\section{Acknowledgments}

The work of L. H He and Z. Tan was supported by the National Natural Science Foundation of China (11531010, 11726023).

\section{References}

[1] A. P. Calderón, "On an inverse boundary value problem," in Seminar on Numerical Analysis and Its Applications to Continuum Physics, pp. 65-73, Soc. Brasileira de Mathemática, Rio de Janeiro, 1980.

[2] G. Uhlmann and J.-N. Wang, "Reconstructing discontinuities using complex geometrical optics solutions," SIAM Journal on Applied Mathematics, vol. 68, no. 4, pp. 1026-1044, 2008.

[3] K. Krupchyk and G. Uhlmann, "Uniqueness in an inverse boundary problem for a magnetic schrödinger operator with a bounded magnetic potential," Communications in Mathematical Physics, vol. 327, no. 3, pp. 993-1009, 2014.

[4] G. Uhlmann, "Inverse problems: seeing the unseen," Bulletin of Mathematical Sciences, vol. 4, no. 2, pp. 209-279, 2014.

[5] J. Sylvester and G. Uhlmann, "A global uniqueness theorem for an inverse boundary value problem," The Annals of Mathematics, vol. 125, no. 1, pp. 153-169, 1987.

[6] K. Krupchyk and G. Uhlmann, "Inverse boundary problems for polyharmonic operators with unbounded potentials," Journal of Spectral Theory, vol. 6, no. 1, pp. 145-183, 2016.

[7] O. Y. Imanuvilov and M. Yamamoto, "Global uniqueness in inverse boundary value problems for Navier-Stokes equations and Lamé system in two dimensions," Inverse Problems, vol. 31, article 035004, 2015.

[8] T. Ghosh, Y. Linb, and J. Xiao, "The calderón problem for variable coefficients nonlocal elliptic operators," Communications in Partial Differential Equations, vol. 42, no. 12, pp. 19231961, 2017.

[9] H. Heck, X. Li, and J.-N. Wang, "Identification of viscosity in an incompressible fluid," Indiana University Mathematics Journal, vol. 56, pp. 2489-2510, 2006.

[10] G. Eskin and J. Ralston, "On the inverse boundary value problem for linear isotropic elasticity," Inverse Problems, vol. 18, pp. 907-921, 2002.

[11] K. Astala and L. Païvärinta, "Calderón's inverse conductivity problem in the plane," Annals of Mathematics, vol. 163, no. 1, pp. 265-299, 2006.

[12] R. Lai, G. Uhlmann, and J.-N. Wang, "Inverse boundary value problem for the Stokes and the Navier-Stokes equations in the plane," Archive for Rational Mechanics and Analysis, vol. 215, no. 3, pp. 811-829, 2015.

[13] K. Krupchyk and G. Uhlmann, "Inverse problems for magnetic Schrödinger operators in transversally anisotropic geometries," Communications in Mathematical Physics, vol. 361, no. 2, pp. 525-582, 2018.

[14] X. Li and J.-N. Wang, "Determination of viscosity in the stationary Navier-Stokes equations," Journal of Differential Equations, vol. 242, no. 1, pp. 24-39, 2007. 
[15] R. P. Agarwal, S. Gala, and M. A. Ragusa, "A regularity criterion in weak spaces to Boussinesq equations," Mathematics, vol. 8, no. 6, p. 920, 2020.

[16] S. Benbernou, S. Gala, and M. A. Ragusa, "On the regularity criteria for the 3D magnetohydrodynamic equations via two components in terms ofBMOspace," Mathematical Methods in the Applied Sciences, vol. 37, no. 15, pp. 2320-2325, 2014.

[17] V. Isakov, "On uniqueness in inverse problems for semilinear parabolic equations," Archive for Rational Mechanics and Analysis, vol. 124, no. 1, pp. 1-12, 1993.

[18] J. Gerbeau, C. Bris, and T. Lelievre, Mathematical Method for the Magnetohynamics of Liquid Metalls, Oxford university press, 2006.

[19] R. Temam, Navier-Stokes Equaions: Theory and Numerical Analysis, American Mathematical Soc., North-Holland, Amsterdam, 1977.

[20] G. Eskin, "Global uniqueness in the inverse scattering problem for the Schrödinger operator with external Yang-Mills potentials," Communications in Mathematical Physics, vol. 222, pp. 503-531, 2001. 